Pacific Journal of Mathematics

ON A PROBLEM OF GAUSS-KUZMIN TYPE FOR CONTINUED
FRACTION WITH ODD PARTIAL QUOTIENTS 


\section{ON A PROBLEM OF GAUSS-KUZMIN TYPE FOR CONTINUED FRACTION WITH ODD PARTIAL QUOTIENTS}

\section{Sofia KalPaZidou}

Let $x$ be a number of the unit interval. Then $x$ may be written in a unique way as a continued fraction

$$
x=1 /\left(\alpha_{1}(x)+\varepsilon_{1}(x) /\left(\alpha_{2}(x)+\varepsilon_{2}(x) /\left(\alpha_{3}(x)+\cdots\right)\right)\right)
$$

where $\varepsilon_{n} \in\{-1,1\}, \alpha_{n} \geq 1, \alpha_{n} \equiv 1(\bmod 2)$ and $\alpha_{n}+\varepsilon_{n}>1$. Using the ergodic behaviour of a certain homogeneous random system with complete connections we solve a variant of Gauss-Kuzmin problem for the above expansion.

1. Introduction. We define continued fraction with odd partial quotients as follows. Let us partition the unit interval into $\left(\frac{1}{2 k}, \frac{1}{2 k-1}\right], k=1,2, \ldots, \quad$ and $\left(\frac{1}{2 k-1}, \frac{1}{2 k-2}\right], \quad k=2,3, \ldots$ and define the transformation $T:[0,1] \rightarrow[0,1]$ by

$$
T x=e\left(\frac{1}{x}-(2 k-1)\right)
$$

where

$$
e=1 \quad \text { if } x \in\left(\frac{1}{2 k}, \frac{1}{2 k-1}\right]
$$

and

$$
e=-1 \quad \text { if } x \in\left(\frac{1}{2 k-1}, \frac{1}{2 k-2}\right]
$$

We arrive at

$$
x=\frac{1}{2 k-1+e(T x)}
$$

and therefore the map $T$ generates a continued fraction

$$
\begin{aligned}
x & =\frac{1}{\alpha_{1}(x)+\varepsilon_{1}(x) /\left(\alpha_{2}(x)+\varepsilon_{2}(x) /\left(\alpha_{3}(x)+\cdots\right)\right)} \\
& =\left[\begin{array}{c}
1, \varepsilon_{1}(x), \varepsilon_{2}(x), \ldots \\
\alpha_{1}(x), \alpha_{2}(x), \alpha_{3}(x), \ldots
\end{array}\right]
\end{aligned}
$$


where $\varepsilon_{n} \in\{-1,1\}, \alpha_{n} \geq 1, \alpha_{n} \equiv 1(\bmod 2)$ and $\alpha_{n}+\varepsilon_{n}>1$. The expression (1.1) is called the continued fraction with odd partial quotients expansion of $x$.

Let us denote

$$
r_{n}=\alpha_{n}+\left[\begin{array}{c}
\varepsilon_{n+1}, \ldots \\
\alpha_{n+1}, \ldots
\end{array}\right], \quad n=1,2, \ldots
$$

The purpose of this paper is to find the limit

$$
\lim _{n \rightarrow \infty} \mu\left(r_{n}>t\right)=l
$$

for a given nonatomic measure $\mu$ on the $\sigma$-algebra of the Borel sets of $[0,1]$ and to estimate the error $\mu\left(r_{n}>t\right)-l$. This is the variant of Gauss-Kuzmin problem for the continued fraction with odd partial quotients expansion. For solving of the above problem we shall use the approach of the random system with complete connections.

NOTATION.

$N^{*}=\{1,2,3, \ldots\}$,

$N=\{0,1,2, \ldots\}$,

$R=$ the set of real numbers,

$[a]=$ the integral part of $a \in R$,

$I_{A}=$ the characteristic function of $A$,

$G=(\sqrt{5}+1) / 2$,

$\mathscr{B}_{[0,1]}=$ the $\sigma$-algebra of the Borel sets of $[0,1]$,

$\mathscr{P}(X)=$ the power set of $X$,

$\left(X, \mathscr{X}^{r}\right)=$ the $n$-fold product measurable space of $(X, \mathscr{X})$.

\section{Preliminaries.}

Definition 2.1. A quadruple $\{(W, \mathscr{W}),(X, \mathscr{X}), u, P\}$ is named a homogeneous random system with complete connections (RSCC) if

(i) $(W, \mathscr{W})$ and $(X, \mathscr{X})$ are arbitrary measurable spaces;

(ii) $u$ : $W \times X \rightarrow W$ is a $(\mathscr{W} \otimes \mathscr{X}, \mathscr{W})$-measurable function;

(iii) $P$ is a transition probability function from $(W, \mathscr{W})$ to $(X, \mathscr{X})$.

Next, denote the element $\left(x_{1}, \ldots, x_{n}\right) \in X^{n}$ by $x^{(n)}$.

Definition 2.2. The functions $u^{(n)}: W \times X^{\mathrm{n}} \rightarrow W, n \in N^{*}$, are defined as follows:

$$
u^{(n+1)}\left(w, x^{(n+1)}\right)= \begin{cases}u(w, x), & \text { if } n=0 \\ u\left(u^{(n)}\left(w, x^{(n)}\right), x_{n+1}\right), & \text { if } n \geq 1 .\end{cases}
$$

Convention. We shall write $w x^{(n)}$ instead of $u^{(n)}\left(w, x^{(n)}\right)$. 
Definition 2.3. The transition probability functions $P_{r}, r \in N^{*}$, are defined by

$P_{r}(w, A)=\left\{\begin{aligned} P(w, A), & \text { if } r=1 \\ \sum_{x_{1} \in X} P\left(w, x_{1}\right) \sum_{x_{2} \in X} P\left(w x_{1}, x_{2}\right) \cdots \sum_{x_{r} \in X} P\left(w x^{(r-1)}, x_{r}\right) I_{A}\left(x^{(r)}\right), & \text { if } r>1,\end{aligned}\right.$

for any $w \in W, r \in N^{*}$ and $A \in \mathscr{X}^{r}$.

Definition 2.4. Assume that $X^{0} \times A=A$. Then we define

$$
P_{r}^{n}(w, A)=P_{n+r-1}\left(w, X^{n-1} \times A\right),
$$

for any $w \in W, n, r \in N^{*}$ and $A \in \mathscr{X}^{r}$.

THEOREM 2.5. (Existence theorem.) Let $\{(W, \mathscr{W}),(X, \mathscr{X}), u, P\}$ be a homogeneous $R S C C$ and let $w_{0} \in W$. Then there exist a probability space $\left(\Omega, \mathscr{K}, P_{w_{0}}\right)$ and two chains of random variables $\left(\xi_{n}\right)_{n \in N^{*}}$ and $(\zeta)_{n \in N}$ defined on $\Omega$ with values in $X$ and $W$ respectively, such that

(i)(a) $P_{w_{0}}\left(\left(\xi_{n}, \ldots, \xi_{n+r-1}\right) \in A\right)=P_{r}^{n}\left(w_{0}, A\right)$,

(b) $P_{w_{0}}\left(\left(\xi_{n+m}, \ldots, \xi_{n+m+r-1}\right) \in A \mid \xi^{(n)}\right)=P_{r}^{m}\left(w_{0} \xi^{(n)}, A\right), P_{w_{0}}-$ a.e.

(c) $P_{w_{0}}\left(\left(\xi_{n+m}, \ldots, \xi_{n+m+r-1}\right) \in A \mid \xi^{(n)}, \zeta^{(n)}\right)=P_{r}^{m}\left(\zeta_{n}, A\right), \quad P_{w_{0}}-$ a.e. for any $n, m, r \in N^{*}$ and $A \in \mathscr{X}^{r}$, where $\xi^{(n)}, \zeta^{(n)}$ denote the random vectors $\left(\xi_{1}, \ldots, \xi_{n}\right)$ and $\left(\zeta_{1}, \ldots, \zeta_{n}\right)$ respectively.

(ii) $\left(\zeta_{n}\right)_{n \in N}$ is a homogeneous Markov chain with initial distribution concentrated in $w_{0}$ and with the transition operator $U$ defined by

$$
U f(w)=\sum_{x \in X} P(w, x) f(w x)
$$

for any f real $W$-measurable and bounded function.

This theorem is proved by Iosifescu [2].

REMARK. (i) Letting $m=r=1$ in (i)b we obtain

$$
P_{w_{0}}\left(\xi_{n+1} \in A \mid \xi^{(n)}\right)=P\left(w_{0} \xi^{(n)}, A\right), \quad P_{w_{0}} \text {-a.e. }
$$

that is the conditioned distribution of $\xi_{n+1}$ by the past depends actually by this, through $u^{(n)}$. This fact justifies the name of chain of infinite order or chain with complete connections used for $\left(\xi_{n}\right)_{n \in N}$. 
(ii) On account of (2.1) we have

$$
U^{n} f(w)=\sum_{x^{(n)} \in X^{n}} P_{n}\left(w, x^{(n)}\right) f\left(w x^{(n)}\right), \quad n \in N^{*}
$$

for any $f$ real $\mathscr{W}$-measurable and bounded function.

(iii) The transition probability function of the Markov chain $\left(\zeta_{n}\right)_{n \in N^{*}}$ is

$$
Q(w, A)=\sum_{x \in X} P(w, x) I_{A}(w x)=P\left(w, A_{w}\right),
$$

where $A_{u^{\prime}}=\{x \in X: w x \in A\}, w \in W$. It follows that the transition probability after $n$ paths of the Markov chain $\left(\zeta_{n}\right)_{n \in N}$ is

$$
Q^{n}(w, A)=P_{n}\left(w, A_{w}^{(n)}\right)
$$

where $A_{n^{\prime}}^{(n)}=\left\{x^{(n)}: w x^{(n)} \in A\right\}$.

2.6. Let $Q_{n}$ be the transition probability function defined by

$$
Q_{n}(w, A)=n^{-1} \sum_{k=1}^{n} Q^{k}(w, A)
$$

and let $U_{n}$ be the Markov operator associated with $Q_{n}$. Next, denote $L(W)$ the space of all real Lipschitz functions defined on $W$ and assume that $(L(W),\|\cdot\|)$ is a Banach space with respect to a norm $\|\cdot\|$.

(i) If there exists a linear bounded operator $U^{\infty}$ from $L(W)$ to $L(W)$ such that

$$
\lim _{n \rightarrow \infty}\left\|U_{n} f-U^{\infty} f\right\|=0
$$

for any $f \in L(W)$ with $\|f\|=1$, we say $U$ ordered.

(ii) If

$$
\lim _{n \rightarrow \infty}\left\|U^{n} f-U^{\infty} f\right\|=0
$$

for any $f \in L(W)$ with $\|f\|=1$, we say $U$ aperiodic.

(iii) If $U$ is ordered and $U^{\infty}(L(W))$ is one-dimensional space, it is named ergodic with respect to $L(W)$.

(iv) If $U$ is ergodic and aperiodic, it is named regular with respect to $L(W)$ and the corresponding Markov chain has the same name.

Definition 2.7. If $\{(W, \mathscr{W}),(X, \mathscr{X}), u, P\}$ is a RSCC which satisfies the properties

(i) $(W, d)$ is a metric separable space; 
(ii) $r_{1}<\infty$, where

$$
r_{k}=\sup _{w^{\prime} \neq w^{\prime \prime}} \sum_{X^{k}} P_{k}\left(w, x^{(k)}\right) \frac{d\left(w^{\prime} x^{(k)}, w^{\prime \prime} x^{(n)}\right)}{d\left(w^{\prime}, w^{\prime \prime}\right)}, \quad k \in N^{*} ;
$$

(iii) $R_{1}<\infty$, where

$$
R_{1}=\sup _{A \in \mathscr{C}} \sup _{w^{\prime} \neq w^{\prime \prime}} \frac{\left|P\left(w^{\prime}, A\right)-P\left(w^{\prime \prime}, A\right)\right|}{d\left(w^{\prime}, w^{\prime \prime}\right)}
$$

(iv) there exists $k \in N^{*}$ such that $r_{k}<1$, it is named RSCC with contraction.

This definition is due to M. F. Norman [3].

THEOREM 2.8. Let $(W, d)$ be a compact space and $\{(W, \mathscr{W})$, $(X, \mathscr{X}), u, P\}$ be a RSCC with contraction.

The Markov chain associated to the RSCC is regular, if and only if, there exists a point $\tilde{w} \in W$ such that

$$
\lim _{n \rightarrow \infty} d\left(\sigma_{n}(\tilde{w}), w\right)=0,
$$

for any $w \in W$, where $\sigma_{n}(w)=\operatorname{supp} Q^{n}(w, \cdot)(\operatorname{supp} \mu$ denotes the support of the measure $\mu$ ).

LEMMA 2.9. We have

$$
\sigma_{m+n}(w)=\overline{\bigcup_{w^{\prime} \in \sigma_{m}(w)} \sigma_{n}\left(w^{\prime}\right)}
$$

for any $m, n \in N, w \in W$ (the line designates the topological aderence).

Theorem 2.8 and Lemma 2.9 are due to Iosifescu [1].

Definition 2.10. Let $\{(W, \mathscr{W}),(X, \mathscr{X}) u, P\}$ be a RSCC. The RSCC is called uniformly ergodic if for any $r \in N^{*}$ there exists a probability $P_{r}^{\infty}$ on $\mathscr{X}^{r}$ such that $\lim \varepsilon_{n}=0$, as $n \rightarrow \infty$, where

$$
\varepsilon_{n}=\sup _{\substack{w \in W, r \subseteq N^{*} \\ A \in \mathscr{X}^{r}}}\left|P_{r}^{n}(w, A)-P_{r}^{\infty}(A)\right|
$$

THEOREM 2.11. Let $(W, d)$ be a compact space. If the $\mathrm{RSCC}\{(W, \mathscr{W})$, $(X, \mathscr{X}), u, P\}$ with contraction has regular associated Markov chain, then it is uniform ergodic.

This result one can find in [1]. 
3. The Gauss-Kuzmin type equation. Let $\mu$ be a nonatomic measure on $\mathscr{B}_{[0,1]}$ and define

$$
F_{n}(w)=F_{n}(w, \mu)=\mu\left(r_{n+1}^{-1}<w\right), \quad n \in N, w \in[0,1] .
$$

Clearly $F_{0}(w)=\mu([0, w])$.

Proposition 3.2. (The Gauss-Kuzmin type equation) $F_{n}, n \in N$, satisfy the relation

$$
F_{n+1}(w)=\sum_{\substack{(k, \varepsilon) \\ k \equiv 1(\bmod 2) \\|\varepsilon|=1, k+\varepsilon>1}} \varepsilon\left(F_{n}\left(\frac{1}{k}\right)-F_{n}\left(\frac{1}{k+\varepsilon w}\right)\right), \quad w \in[0,1] .
$$

Proof. We start from the relation

$$
r_{n+1}=\alpha_{n+1}+\frac{\varepsilon_{n+1}}{r_{n+2}}
$$

Thus

$$
\begin{aligned}
F_{n+1}(w)= & \mu\left(r_{n+2}^{-1}<w, \varepsilon_{n+1}=1\right)+\mu\left(r_{n+1}^{-1}<w, \varepsilon_{n+1}=-1\right) \\
= & \sum_{k=1(\bmod 2)} \mu\left((k+w)^{-1}<r_{n+1}^{-1}<k^{-1}\right) \\
& +\sum_{k \equiv \sum_{k \neq 1}(\bmod 2)} \mu\left(k^{-1}<r_{n+1}^{-1}<(k-w)^{-1}\right) \\
= & \sum_{(k, \varepsilon)} \varepsilon\left(F_{n}\left(\frac{1}{k}\right)-F_{n}\left(\frac{1}{k+\varepsilon w}\right)\right) .
\end{aligned}
$$

and this completes the proof.

Further, suppose that $F_{0}^{\prime}$ exists and it is bounded ( $\mu$ has bounded density). By induction we obtain that $F_{n}^{\prime}$ exists and it is bounded too for any $n \in N^{*}$. Deriving the Gauss-Kuzmin type equation we arrive at

$$
F_{n+1}^{\prime}(w)=\sum_{(k, \varepsilon)} \frac{1}{(k+\varepsilon w)^{2}} F_{n}^{\prime}\left(\frac{1}{k+\varepsilon w}\right) .
$$

Let us denote for $\rho(w)=(G-1+w)^{-1}-(-G-1+w)^{-1}, w \in$ $[0,1]$ and $n \in N$

$$
f_{n}(w)=F_{n}^{\prime}(w) / \rho(w)
$$


Then (3.1) becomes

$$
\begin{aligned}
& f_{n+1}(w)=\left(G^{2}-(1-w)^{2}\right) \\
& \quad \times \sum_{(k, \varepsilon)} \frac{1}{((G-1)(k+\varepsilon w)+1)((G+1)(k+\varepsilon w)-1)} f_{n}\left(\frac{1}{k+\varepsilon w}\right) .
\end{aligned}
$$

Now, we prove

\section{Proposition 3.3. The function}

$$
P(w,(k, \varepsilon))=\frac{G^{2}-(1-w)^{2}}{((G-1)(k+\varepsilon w)+1)((G+1)(k+\varepsilon w)-1)}
$$

defines a transition probability function from $\left([0,1], \mathscr{B}_{[0,1]}\right)$ to $(X, \mathscr{P}(X))$ where

$$
X=\{(k, \varepsilon): k \geq 1, k \equiv 1(\bmod 2),|\varepsilon|=1, k+\varepsilon>1\} .
$$

Proof. We must verify that

$$
\sum_{(k, \varepsilon)} P(w,(k, \varepsilon))=1 .
$$

Indeed, noting that $(G-1)^{-1}=G$ and $(G+1)^{-1}=-G+2$, we have

$$
\begin{aligned}
& \sum_{(k, \varepsilon)} \frac{G^{2}-(1-w)^{2}}{((G-1)(k+\varepsilon w)+1)((G+1)(k+\varepsilon w)-1)} \\
& =\frac{G^{2}-(1-w)^{2}}{G^{2}-1}\left(\sum_{k=1,3, \ldots} \frac{1}{(k+w+G)(k+w+G-2)}\right. \\
& \left.\quad+\sum_{k=3,5, \ldots} \frac{1}{(k-w+G)(k-w+G-w)}\right) \\
& =\frac{G^{2}-(1-w)^{2}}{2 G}\left(\sum_{k=1,3, \ldots}\left(\frac{1}{k-2+w+G}-\frac{1}{k+w+G}\right)\right. \\
& \left.\quad+\sum_{k=3,5, \ldots}\left(\frac{1}{k-2-w+G}-\frac{1}{k-w+G}\right)\right) \\
& =\frac{G^{2}-(1-w)^{2}}{2 G}\left(\frac{1}{G-(1-w)}+\frac{1}{G+(1-w)}\right)=1
\end{aligned}
$$

that is the desired result. 
Now, we can define a random system with complete connections as follows.

$$
\{(W, \mathscr{W}),(X, \mathscr{X}), u, P\}
$$

where

$$
\begin{gathered}
W=[0,1], \quad \mathscr{W}=\mathscr{B}, \\
X=\{(k, \varepsilon): k \geq 1, k \equiv 1(\bmod 2),|\varepsilon|=1, k+\varepsilon>1\}, \\
\mathscr{X}=\mathscr{P}(X), \quad u(w,(k, \varepsilon))=\frac{1}{k+\varepsilon w} \\
P(w,(k, \varepsilon))=\frac{G^{2}-(1-w)^{2}}{((G-1)(k+\varepsilon w)+1)((G+1)(k+\varepsilon w)-1)} .
\end{gathered}
$$

4. The ergodic behaviour of the RSCC. In this section we study the ergodic behaviour of RSCC (3.2) in order to solve a Gauss-Kuzmin type theorem.

In what follows we shall introduce the norm $\|\cdot\|_{L}$ defined by

$$
\|f\|_{L}=\sup _{w \in W}|f(w)|+\sup _{w^{\prime} \neq w^{\prime \prime}} \frac{\left|f\left(w^{\prime}\right)-f\left(w^{\prime \prime}\right)\right|}{\left|w^{\prime}-w^{\prime \prime}\right|}, \quad f \in L(W) .
$$

Then $\left(L(W),\|\cdot\|_{L}\right)$ is a Banach algebra.

Proposition 4.1. RSCC (3.2) is a RSCC with contraction and its associated Markov operator $U$ is regular with respect to $L(W)$.

Proof. We have

$$
\begin{gathered}
\frac{d P}{d w}=\frac{2(1-w)((G-1)(x+\varepsilon w)+1)((G+1)(x+\varepsilon w)-1)}{((G-1)(k+\varepsilon w)+1)^{2}((G+1)(k+\varepsilon w)-1)^{2}} \\
-\frac{2 \varepsilon\left(G^{2}-(1-w)^{2}\right)\left(\left(G^{2}-1\right)(x+\varepsilon w)+1\right)}{((G-1)(k+\varepsilon w)+1)^{2}((G+1)(k+\varepsilon w)-1)^{2}}, \\
\frac{d u}{d w}=\frac{\varepsilon}{(k+\varepsilon w)^{2}} .
\end{gathered}
$$

Therefore

$$
\begin{gathered}
\sup _{w \in W,(x, \varepsilon) \in X}\left|\frac{d}{d w} P(w,(x, \varepsilon))\right|<\infty, \\
\sup _{w \in W}\left|\frac{d}{d w} u(w,(x, \varepsilon))\right|<\frac{1}{(x-1)^{2}}, \quad k=3,5, \ldots
\end{gathered}
$$


It follows that $R_{1}<\infty$ and $r_{1}<1$, that is, (3.2) is a SALC with contraction.

To prove the regularity of $U$ with respect to $L(W)$, define the recurrence relation $w_{n+1}=\left(w_{n}+1\right)^{-1}, n \in N$, with $w_{0}=w$. Clearly $w_{n+1}$ $\in \sigma\left(w_{n}\right)$. Then using Lemma 2.9 and by induction we obtain $w_{n} \in \sigma_{n}(w)$, $n \in N^{*}$. Because $w_{n}$ tends to $G-1$ as $n \rightarrow \infty$, for every $w \in[0,1]$, then

$$
\left|\sigma_{n}(w), G-1\right| \leq\left|w_{n}-G+1\right| \rightarrow 0
$$

as $n \rightarrow \infty$. The regularity of $U$ with respect to $L(W)$ follows from Theorem 2.8. and the proof is completed.

Now, by virtue of Theorem 2.11, RSCC (3.2) is uniformly ergodic. Moreover, Theorem 2.1.57 of Iosifescu-Theodorescu [2], implies that $Q^{n}(\cdot, \cdot)$ converges uniformly to a probability $Q^{\infty}$ and there exist two positive constants $q<1$ and $c$ such that

$$
\left\|U^{n} f-U^{\infty} f\right\|_{L} \leq c q^{n}
$$

for all $n \in N^{*}, f \in L(W)$, where

$$
U^{\infty} f=\int_{W} f(w) Q^{\infty}(d w)
$$

Further, by virtue of Lemma 2.1.58 of Iosifescu-Theodorescu [2], $U$ has no eigenvalues of modulus 1 other than 1 . Then, taking into account Proposition 2.1.6 of [2] the adjoint of the operator $U$ with the transition probability function $Q$ has the only eigenvector the measure $Q^{\infty}$, that is

$$
\int_{0}^{1} Q(w, B) Q^{\infty}(d w)=Q^{\infty}(B)
$$

for all the Borel sets $B$ of $[0,1]$.

Generally, the form of $Q^{\infty}$ cannot be identified but in our case this is possible as we shall show below

Proposition 4.2. The probability $Q^{\infty}$ has the density

$$
\rho(w)=\frac{1}{w+G-1}-\frac{1}{w-G-1}, \quad w \in[0,1]
$$


Proof. By virtue of uniqueness of $Q^{\infty}$ we have to prove the equality (4.3) where

$$
Q(w, B)=\sum_{\substack{(x, \varepsilon) \in X \\(x+\varepsilon w)^{-1} \in B}} P(w,(x, \varepsilon)), \quad w \in[0,1], B \in \mathscr{B}_{[0,1]} .
$$

Since the intervals $[0, u] \subset[0,1]$ generate $\mathscr{B}_{[0,1]}$, it suffices to verify the equality (4.3) only for $B=[0, u], 0<u \leq 1$. First, we consider that $\left[u^{-1}\right]$ is even. Then

$$
\begin{aligned}
& \int_{0}^{1} Q(w,[0, u)) \rho(w) d w \\
& \begin{array}{l}
=\int_{0}^{1}\left(\sum_{\substack{k=1,3, \ldots \\
k>\left[u^{-1}-w\right]}} P(w,(k, 1))+\sum_{\substack{k=3,5, \ldots \\
k>\left[u^{-1}\right]}} P(w,(k,-1))\right) \rho(w) d w \\
=\int_{0}^{1} \frac{G^{2}-(1-w)^{2}}{2\left(G^{2}-1\right)}
\end{array} \\
& \times\left(\sum_{k=\left[u^{-1}\right]+1}^{\infty}\left(\frac{1}{k-2+w+G}-\frac{1}{k+w+G}\right)\right) \rho(w) d w \\
& +\int_{0}^{1+\left[u^{-1}\right]-u^{-1}} \frac{G^{2}-(1-w)^{2}}{2 G} \\
& \times\left(\sum_{k=\left[u^{-1}\right]+1}^{\infty}\left(\frac{1}{k-2+w+G}-\frac{1}{k+w+G}\right) \rho(w) d w\right. \\
& +\int_{1+\left[u^{-1}\right]-u^{-1}}^{1} \frac{G^{2}-(1-w)^{2}}{2 G} \\
& \times\left(\sum_{k=\left[u^{-1}\right]+3}^{\infty}\left(\frac{1}{k-2-w+G}-\frac{1}{k-w+G}\right) \rho(w) d w\right. \\
& =\log \frac{G+\left[u^{-1}\right]}{G+\left[u^{-1}\right]-1} \cdot \frac{G+\left[u^{-1}\right]-1}{G+u^{-1}-2} \cdot \frac{G+u^{-1}}{G+\left[u^{-1}\right]} \\
& =\log \frac{(G+1)(G-1+u)}{(G-1)(G+1-u)}=\int_{0}^{u} \rho(w) d w .
\end{aligned}
$$


Analogously if $\left[u^{-1}\right]$ is odd we have

$$
\begin{aligned}
\int_{0}^{1} Q(w,[0, u)) \rho(w) d w \\
=\int_{0}^{u^{-1}-\left[u^{-1}\right]} \frac{G^{2}-(1-w)^{2}}{2 G} \cdot \frac{\rho(w)}{\left[u^{-1}\right]+w+G} d w \\
\quad+\int_{u^{-1}-\left[u^{-1}\right]}^{1} \frac{G^{2}-(1-w)^{2}}{2 G} \cdot \frac{\rho(w)}{\left[u^{-1}\right]-2+w+G} d w \\
\quad+\int_{0}^{1} \frac{G^{2}-(1-w)^{2}}{2 G} \cdot \frac{\rho(w)}{\left[u^{-1}\right]-w+G} d w \\
=\log \frac{u^{-1}+G}{\left[u^{-1}\right]+G} \cdot \frac{\left[u^{-1}\right]+G-1}{u^{-1}+G-1} \cdot \frac{\left[u^{-1}\right]+G}{\left[u^{-1}\right]+G-1} \\
=\log \frac{(G+1)(G-1+u)}{(G-1)(G+1-u)}=\int_{0}^{u} \rho(w) d w .
\end{aligned}
$$

5. The Gauss-Kuzmin type theorem. Now, we may determine where $\mu\left(r_{n}>t\right)$ tends as $n \rightarrow \infty$ and give the rate of this convergence.

Proposition 5.1. (The solution of Gauss-Kuzmin type problem.) If the density $F_{0}^{\prime}$ of $\mu$ is a Riemann integrable function, then

$$
\lim _{n \rightarrow \infty} \mu\left(r_{n}>t\right)=\frac{1}{3 \log G} \cdot \log \frac{(G+1)(t(G-1)+1)}{(G-1)(t(G+1)-1)}, \quad t \geq 1 .
$$

If the density $F_{0}^{\prime}$ of $\mu$ is a Lipschitz function, then there exist two positive constants $c$ and $q<1$ such that for all $t \geq 1, n \in N^{*}$

$$
\mu\left(r_{n}>t\right)=\frac{1}{3 \log G}\left(1+\theta q^{n}\right) \log \frac{(G+1)(t(G-1)+1)}{(G-1)(t(G+1)-1)}
$$

where $\theta=\theta(\mu, n, t)$ with $|\theta| \leq c$.

Proof. Let $F_{0}^{\prime}$ be a Lipschitz function. Then $f_{0} \in L(W)$ and by virtue of (4.2)

$$
U^{\infty} f_{0}=\int_{0}^{1} f_{0}(w) Q^{\infty}(d w)=\frac{1}{3 \log G} \int_{0}^{1} F_{0}^{\prime}(w) d w=\frac{1}{3 \log G} .
$$

According to (4.1) there exist two constants $c$ and $q<1$ such that 


$$
U^{n} f_{0}=U^{\infty} f_{0}+T^{n} f_{0}, \quad n \in N^{*},
$$

with $\left\|T^{n} f_{0}\right\|_{L} \leq c q^{n}$.

Further, consider $C[0,1]$ the metric space of real continuous functions defined on $[0,1]$ with the norm $|\cdot|=\sup |\cdot|$. Since $L([0,1])$ is a dense subset of $C([0,1])$ we have

$$
\lim _{n \rightarrow \infty}\left|T^{n} f_{0}\right|=0
$$

for $f_{0} \in C([0,1])$. Therefore (5.1) is valid for measurable $f_{0}$ which is $Q^{\infty}$-almost surely continuous, that is for Riemann integrable $f_{0}$. Thus

$$
\begin{aligned}
\lim _{n \rightarrow \infty} \mu\left(r_{n}>t\right) & =\lim _{n \rightarrow \infty} F_{n-1}\left(\frac{1}{t}\right) \\
& =\lim _{n \rightarrow \infty} \int_{0}^{1 / t} U^{n-1} f_{0}(u) \rho(u) d w \\
& =\frac{1}{3 \log G} \int_{0}^{1 / t} \rho(w) d w
\end{aligned}
$$

and the desired result follows.

\section{REFERENCES}

[1] S. Grigorescu and M. Iosifescu, Dependence with Complete Connections and Applications, Ed. St. Encicl., Bucharest, 1982.

[2] M. Iosifescu and R. Theodorescu, Random Processes and Learning, Springer-Verlag, Berlin, 1969.

[3] M. F. Norman, Markov Processes and Learning Models, Academic Press, New York, 1972.

[4] F. Schweiger, Continued fractions with odd and even partial quotients, Arbeitsbericht Math. Instit. der Univ. Salzburg, 4 (1982), 59-70.

Received October 5, 1984.

ARISTOTLE UNIVERSITY

THESSALONIKI, GREECE 


\section{PACIFIC JOURNAL OF MATHEMATICS EDITORS}

V. S. VARADARAJAN

(Managing Editor)

University of California

Los Angeles, CA 90024

HERBERT CLEMENS

University of Utah

Salt Lake City, UT 84112

R. FINN

Stanford University

Stanford, CA 94305
HERMANN FLASCHKA

University of Arizona

Tucson, AZ 85721

RAMESH A. GANGOLLI

University of Washington

Seattle, WA 98195

VAUGHAN F. R. JONES

University of California

Berkeley, CA 94720

ROBION KIRBY

University of California

Berkeley, CA 94720
C. C. MOORE

University of California

Berkeley, CA 94720

H. SAMELSON

Stanford University

Stanford, CA 94305

HAROLD STARK

University of California, San Diego

La Jolla, CA 92093

\section{ASSOCIATE EDITORS}

R. ARENS

E. F. BECKENBACH

B. H. NEUMANN (1906-1982)
F. WOLF

K. YOSHIDA

\section{SUPPORTING INSTITUTIONS}

UNIVERSITY OF ARIZONA

UNIVERSITY OF BRITISH COLUMBIA

CALIFORNIA INSTITUTE OF TECHNOLOGY

UNIVERSITY OF CALIFORNIA

MONTANA STATE UNIVERSITY

UNIVERSITY OF NEVADA, RENO

NEW MEXICO STATE UNIVERSITY

OREGON STATE UNIVERSITY
UNIVERSITY OF OREGON UNIVERSITY OF SOUTHERN CALIFORNIA

STANFORD UNIVERSITY

UNIVERSITY OF HAWAII

UNIVERSITY OF TOKYO

UNIVERSITY OF UTAH

WASHINGTON STATE UNIVERSITY

UNIVERSITY OF WASHINGTON 


\section{Pacific Journal of Mathematics}

\section{Vol. 123, No. $1 \quad$ March, 1986}

Maria Emilia Alonso García, A note on orderings on algebraic varieties $\ldots \ldots 1$

F. S. De Blasi and Józef Myjak, On continuous approximations for

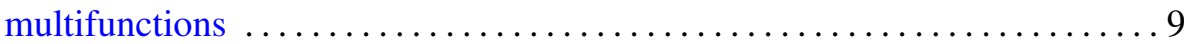

Frank Albert Farris, An intrinsic construction of Fefferman's CR metric . . . 33 Antonio Giambruno, P. Misso and Francisco César Polcino Milies, Derivations with invertible values in rings with involution $\ldots . \ldots . \ldots .47$

Dan Haran and Moshe Jarden, The absolute Galois group of a pseudo real

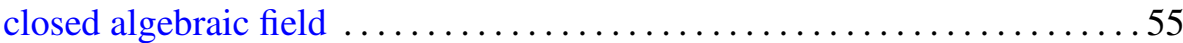

Telemachos E. Hatziafratis, Integral representation formulas on analytic

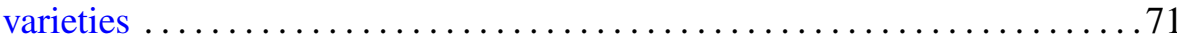

Douglas Austin Hensley, Dirichlet's theorem for the ring of polynomials

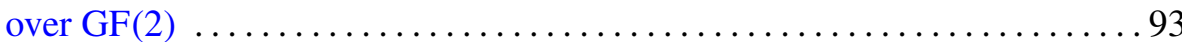

Sofia Kalpazidou, On a problem of Gauss-Kuzmin type for continued

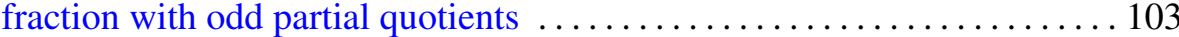

Harvey Bayard Keynes and Mahesh Nerurkar, Ergodicity in affine

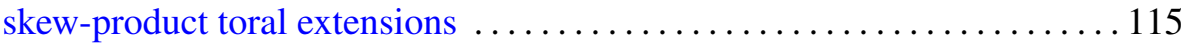

Thomas Landes, Normal structure and the sum-property $\ldots \ldots \ldots \ldots \ldots 127$

Anthony To-Ming Lau and Viktor Losert, Weak*-closed complemented invariant subspaces of $L_{\infty}(G)$ and amenable locally compact groups ...149 Andrew Lelek, Continua of constant distances in span theory . . . . . . . . 161 Dominikus Noll, Sums and products of $B_{r}$ spaces $\ldots \ldots \ldots \ldots \ldots \ldots \ldots \ldots$ Lucimar Nova, Fixed point theorems for some discontinuous operators 189

A. A. S. Perera and Donald Rayl Wilken, On extreme points and support points of the family of starlike functions of order $\alpha$

Massimo A. Picardello, Positive definite functions and $L^{p}$ convolution operators on amalgams ........................

Friedrich Roesler, Squarefree integers in nonlinear sequences ......... 223

Theodore Shifrin, The osculatory behavior of surfaces in $\mathbf{P}^{5}$ 